\title{
STEADY-STATE FORCED VIBRATIONS
}

\author{
R. M. ROSENBERG \\ Department of Applied Mechanics, University of California, Berkeley
}

\begin{abstract}
The following concepts are defined: (i) periodic functions possessing an amplitude, (ii) cosine-like functions, (iii) steady-state forced vibrations. The following theorems are proved:

I. If, in

$$
\begin{gathered}
\ddot{x}+f(x)=P g(t)=P g(t+T), \\
x(0)=X \neq 0, \quad 0<P<\infty, \quad-\infty<t<+\infty,
\end{gathered}
$$

$g(t)$ is cosine-like, a necessary condition for the existence of steady-state forced vibrations is that $f(x)$ be everywhere analytic, odd, monotonely increasing with $x$, and $x f(x)>0,(x \neq 0)$.

II. Response curves of steady-state forced vibrations $T=T(X, P)$ satisfy a first order differential equation that has precisely one singularity at $\left[X=0, T=T_{0}(0)=T(0,0)\right.$; it is a saddle point.
\end{abstract}

The topological portrait of the response curves is constructed.

\section{INTRODUCTION}

THE problem of the so-called "steady-state forced vibrations" is of technical importance largely because of the two phenomena known, respectively, as "resonance" and "dynamic vibration absorption". The former is a vibration of large amplitude, produced by a periodic exciting force of small amplitude, where excitation and response are equiperiodic. Resonance may occur in systems having one, or more than one, degree of freedom. Dynamic vibration absorption can only occur in systems having more than one degree of freedom. It is a vibration of the system such that the motion in one or several (but not all) degrees of freedom vanishes identically while that in the remaining ones is equiperiodic with the excitation and bounded.

The steady-state forced vibration is usually represented by the so-called "response curves", called hereafter R-curves. These are families of one-parameter curves in the frequency-amplitude planes, and the force amplitude is the parameter. Clearly, there are as many such planes as there are degrees of freedom, and each plane contains a family of R-curves. Thus, R-curves are the loci of points relating the frequency of the motion (and, hence, of the force) to the amplitudes of the motion in each of the degrees of freedom: in these diagrams, the force-amplitude is held constant along each curve.

There can be little doubt that the R-curve representation arose from a study of the linear system, excited by a simple harmonic force, and that theory is readily accessible [1]. However, the same representation has also been used in non-linear systems with one $[2-4]$, or with more than one $[5,6]$ degree of freedom. R-curves have the well-known appearance shown for a linear system in Fig. 1(a) and for a non-linear one in Fig. 1(b). In both illustrations, a two-degree-of-freedom system was chosen and both exhibit resonance as well as dynamic vibration absorption. 

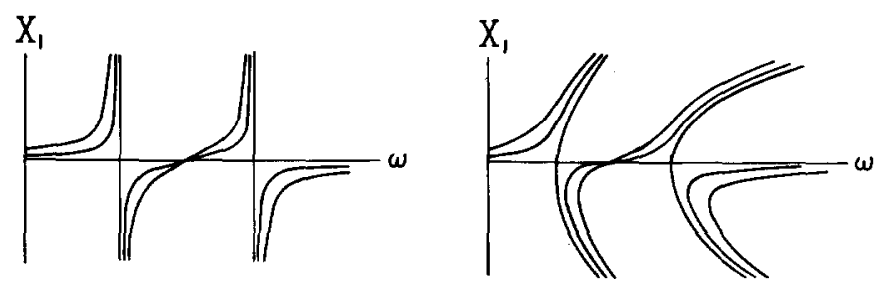

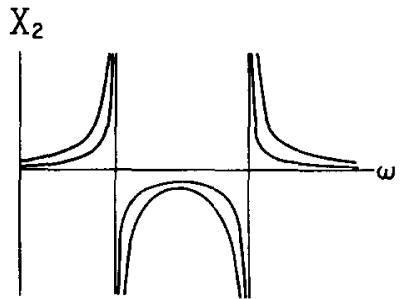

(a) Linear

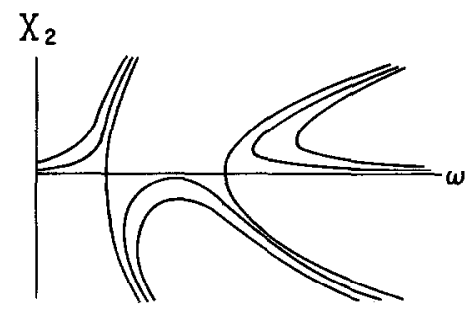

(b) Nontinear

FIG. 1. Response curves.

It is curious that the literature on the subject does not appear to contain any clear definition or critical evaluation of the concept of "amplitude" (of periodic forces or motions) even though it is clear that periodic functions need not have a single "amplitude" in the implicitly understood sense of that word. Hence, when periodic forces have no "amplitude", one cannot use that quantity as a parameter, and when periodic motions have no "amplitude", the frequency-amplitude planes do not exist and the entire concept of R-curves becomes meaningless. Similar remarks apply to the concept of "steady-state forced vibrations" when the system is non-linear.

It is the purpose, in this paper, to give a definition of "amplitude", of "steady-state forced vibrations having amplitudes", and to discuss the response curves of these vibrations by means of the differential equations which $\mathbf{R}$-curves satisfy. One may conjecture from Fig. 1 that these differential equations have singular points lying in the frequency-axis, that only saddle points and star points can occur, and that the former are associated with resonance and the latter with dynamic vibration absorption. In the linear problem, and in some non-linear ones where R-curves are known in closed form, these conjectures have been verified [6-8]. However, where the problem is not soluble in closed form, the above conjecture remains untested.

In this paper, only systems having a single degree of freedom are considered. However, the methods used here are also applicable to systems with many degrees of freedom because steady-state forced vibrations are so-called "vibrations-in-unison", and for a non-linear system vibrating in unison, an $n$-degree-of-freedom system may be decomposed into $n$ systems, each having a single degree of freedom [8].

\section{THE AMPLITUDE}

Consider a fixed point $\mathrm{O}$, and a fixed, simple, smooth curve $\mathrm{C}$, passing through $\mathrm{O}$. Then, if a point $P$ can move along $C$, its position with respect to $O$ may be defined by the arclength $s(t)$ measured from $\mathrm{O}$ to $\mathrm{P}$ (positive to one side of $\mathrm{O}$, and negative to the other). The motion 
of $P$ is periodic of least period $T=2 \pi / \omega$ (where $\omega$ is called the "circular frequency", or simply the "frequency"), if

$$
s(t)=s(t+2 \pi / \omega) .
$$

Since the vibrating point oscillates back and forth along $\mathrm{C}$ there will be instants $t_{j}$ when the value of $s(t)$ is largest or smallest with respect to neighboring $t$. If the velocity exists at these $t_{j}$, it is*

$$
\dot{s}\left(t_{j}\right)=\dot{s}\left(t_{j}+2 \pi / \omega\right)=0, \quad(j=1,2, \ldots) .
$$

Let the values of $s(t)$ at the instants $t_{j}$ be denoted by

$$
s\left(t_{j}\right)=S_{j} .
$$

Then we introduce:

Definition I : A differentiable periodic function $s(t)$ of least period $T$ possesses an amplitude $S$ iff there exist precisely two distinct values $t_{1}$ and $t_{2}$ on $\left(t_{0}, t_{0}+T\right]$ such that

$$
\dot{s}\left(t_{1}\right)=\dot{s}\left(t_{2}\right)=0 \text {. }
$$

The amplitude is then given by

$$
S=\frac{1}{2}\left(S_{1}-S_{2}\right)
$$

where

$$
S_{1}=s\left(t_{1}\right), \quad S_{2}=s\left(t_{2}\right) .
$$
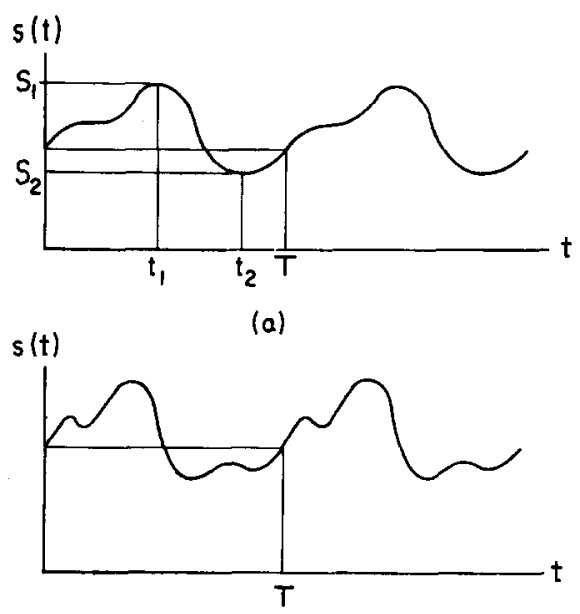

(b)

FIG. 2. Periodic motions (a) possessing an amplitude, (b) not possessing an amplitude.

The reason for defining "amplitude" in this manner is illustrated in Fig. 2. The function shown in the first diagram does possess an amplitude in the sense of Definition I, while that in the second diagram does not. In fact, Definition I assures that $s(t)$ between adjacent maxima consists of two non-cverlapping arcs each of which is monotone, and the method of constructing R-curves depends on this monotonicity.

* Dots denote time-differentiation. 
Definition I may seem unnecessarily restrictive because it excludes non-differentiable, periodic functions. For instance, the saw-tooth curve has no amplitude within Definition I, because there exists no value of $t$ for which its derivative vanishes. While one could readily broaden Definition I to include such functions, we have no need for this generalization because, in our problem, $s(t)$ is always a motion satisfying Newton's second law for all $t$; hence, discontinuities in velocity cannot arise.

It should be emphasized that the steady-state vibration of a linear system might very well be a periodic motion not having an amplitude in the sense of Definition I. The treatment of forced vibrations in the linear system is usually one in which the response does have an amplitude, but this is the result of the choice of a forcing function having an amplitude, not of the linearity of the problem.

Since the properties of the forcing function play an essential role in producing a response with amplitude when the system is linear, one may reasonably expect that the same will also be truc in non-lincar systems.

\section{COSINE-LIKE FUNCTIONS}

Consider a function $s(t)$. We now introduce :

Definition $I I$ : A function $s(t)$ is said to be cosine-like if it has all of the following properties :

(i) $s(t)$ is analytic everywhere in $-\infty<t<+\infty$;

(ii) $s(0)=1, \quad \dot{s}(0)=0$;

(iii) $s(t)=s(t+T) \quad$ where $T=$ const. is the least period of $s$;

(iv) $s(T / 4)=0$;

(v) $s(t)=-s(T / 2-t)$;

(vi) $s(t+\varepsilon)<s(t)$ for $0 \leqslant t<t+\varepsilon \leqslant T / 4$.

Functions $s(t)$ satisfying these conditions are called "cosine-like" because $A \cos 2 \pi t / T$, ( $A=$ const.) belongs to $s(t)$. Properties (i) through (iv) are self-explanatory. Property (v) shows that $s(t)$ has point symmetry with respect to $(s=0, t=T / 4)$; i.e. $s(t)$ in $T / 4 \leqslant t \leqslant T / 2$ may be obtained from $s(t)$ in $0 \leqslant t \leqslant T / 4$ by a sequence of two reflections: one in the line $s=0$, then in the line $t=T / 4$. Also, because of (iv),

$$
\int_{0}^{T / 2} S(t) \mathrm{d} t=0
$$

Property (vi) shows that, with increasing $t, s(t)$ decreases monotonely from $s(0)=1$ to $s(T / 4)=0$; i.e. in $[0, T / 4]$. By property $(v), s(t)$ continues to decrease monotonely until $s(T / 2)=-1$. Thus, $s(t)$ is monotone in $[0, T / 2]$ and in $[T / 2, T]$; in consequence, it has an amplitude in the sense of Definition $I$ and that amplitude is $S=1$.

While cosine-like functions resemble cosine-functions they are, of course, more general. The generalization which is, perhaps, the most significant in the problem of steady-state forced vibrations is that the ratio of two distinct, equiperiodic cosine-like functions satisfying (ii) is in general a function of $t$, while the ratio of two equiperiodic cosine-functions satisfying Property (ii) is a constant. However, the sum of two equiperiodic cosine-like functions satisfying (ii) is always a cosine-like function (except for a constant). 


\section{THE EQUATION OF MOTION}

We consider a system governed by an equation of the form

$$
\ddot{x}+f(x)=P g(t)
$$

where $P>0$ is a constant, and $g(t)$ is cosine-like of period $T$ in the sense of Definition II.

Consider a solution $x(t)$ of $(1)$ which has the initial value

$$
x(0)=X \neq 0
$$

where $X$ may be positive or negative. Then, if $x(t) / X$ is cosine-like of period $T$, it has all the properties usually associated with steady-state forced vibrations of (1). In particular, force and solution arc equiperiodic and in phase if $X>0$, or out of phase if $X<0$. Moreover, both the force $P g(t)$ and the solution $x(t)$ have amplitudes. Thus, we introduce:

Definition $I I I$ : A solution $x(t)$ of $(1)$ is said to be a steady-state forced vibration if $x(t) / X$ is cosine-like of the same period as $g(t)$ where $X=x(0) \neq 0$.

It should be observed that, when $X>0, x(t)$ has an appearance like $g(t)$, but it looks like $-g(t)$ when $X<0$. Moreover, the ratio of force to response is not, in general, a constant. In the linear problem, normally treated [1], that ratio is a constant and, in some treatments of non-linear problems, that ratio was implicitly assumed to be constant; however, it was shown subsequently $[6,9]$, that this assumption is in general unwarranted in the non-linear problem.

We now ask: what must be the structure of (1) such that a steady-state forced vibration can exist? We shall prove:

Theorem I:If $g(t)$ in (1) is cosine-like in the sense of Definition II, then it is necessary for the existence of a steady-state forced vibration of (1) (in the sense of Definition $I I I)$ that $f(x)$ be an analytic, odd function which increases monotonely with $x$ increasing, and for which $x f(x)>0$.

It is obvious that $f(x)$ is analytic. Let us write (1) in the form

$$
f(x)=P g(t)-\ddot{x} \underline{\Delta} h(t) .
$$

Then by Property (i), $h(t)$ is analytic in $t$; hence $f[x(t)]$ is also analytic in $t$, or all timederivatives of $f[x(t)]$ exist. But, these involve all $x$-derivatives of $f$ and all $t$-derivatives of $x$. Since all $t$-derivatives of $x$ and of $h$ exist, all $x$-derivatives of $f$ exist as well.

To show that $f(x)$ is odd, we write (1) for the time

$$
\tau_{1}=T / 2-t
$$

or

$$
\ddot{x}\left(\tau_{1}\right)+f\left[x\left(\tau_{1}\right)\right]=P g\left(\tau_{1}\right) .
$$

But $x(t) / X$ has Property (v); hence, $x(t)$ also has that property: Consequently, the last equation may be written as

$$
-\ddot{x}(t)+f[-x(t)]=-P g(t) .
$$

Adding (1) to this equation, one has

or $f(x)$ is odd.

$$
f[-x(t)]+f[x(t)]=0
$$


To show that $f(x)$ is monotone, we write (1) for the time

$$
\tau_{2}=t+\varepsilon, \quad 0<\tau_{2} \leqslant T / 4
$$

or

$$
\ddot{x}(t+\varepsilon)+f[x(t+\varepsilon)]=P g(t+\varepsilon) .
$$

Then, making use in the above equation of the notation

$$
\begin{aligned}
& x(t+\varepsilon)=x(t)+\Delta x, \\
& g(t+\varepsilon)=g(t)+\Delta g,
\end{aligned}
$$

and subtracting (1) from it we find

$$
f(x+\Delta x)-f(x)=P \Delta g-\Delta \ddot{x} .
$$

We now suppose that $X>0$. [The case $X<0$ need not be considered in view of the fact that $f(x)$ is odd.] For positive $X, x(t) / X$ and $x(t)$ have the same behavior. In particular $\ddot{x}(0)<0$, and $\ddot{x}(t)$ increases monotonely in $0 \leqslant t \leqslant T / 4$; hence, $\Delta \ddot{x}>0$. But, by Property (vi) $\Delta g<0$, and by definition, $P>0$. Consequently,

$$
f(x+\Delta x)-f(x)<0 .
$$

This shows that $f(x)$ is monotone. The fact that it increases with increasing $x$ follows from Property (vi) on $\Delta x$; i.e. $\Delta x<0$ on the interval $[X, 0]$. Thus, the above inequality may be written as

$$
f(x)-f(x-|\Delta x|)>0 .
$$

Hence, $f(x)$ increases with increasing $x$. The last property in Theorem I states that $f(x)$ has the same sign as its argument. We prove it by writing (1) for $t=0$; i.e.

$$
f(X)=P-\ddot{x}(0) .
$$

But, when $X>0, \ddot{x}(0)<0$, or $P-\ddot{x}(0)>0$; hence, $f(X)$ has the same sign as $X$. This completes the proof of Theorem I.

From Theorem I, it is evident that, when seeking R-curves of a system like (1), we must consider as the equation of motion

$$
\ddot{x}+f(x)=P g(t)
$$

where $P$ is a positive constant, $g(t)$ is cosine-like in the sense of Definition II, and $f(x)$ is an analytic, monotone function satisfying

$$
\begin{aligned}
& f(x)=-f(-x), \\
& f(0)=0, \\
& x f(x)>0,(x \neq 0) .
\end{aligned}
$$

It is clear that this system may be very strongly non-linear, and that the forcing function need not be simple harmonic. 


\section{FREQUENCY-AMPLITUDE RELATION}

If (1) has a steady-state solution in the sense of Definition III, its initial values are

$$
x(0)=X \neq 0, \quad \dot{x}(0)=0 .
$$

Thus, the solution must be of the form

$$
x=x(t, X)=x[t+T(X), X]
$$

where we have foreseen the possibility that the period $T$ may be a function of the amplitude $X$. Moreover, since $f(x)$ and $g(t)$ are both analytic, (5) must be a continuous function of the initial value $X$.

Now, it will be observed that (3) and (4) remain unchanged when $t$ is replaced by $-t$; thus, $t=0$ is a line of symmetry for $x(t)$. This permits the construction of $x(t)$ in $[-T / 2$, $T / 2]$ from its behavior in $[0, T / 4]$. From this we deduce that the least time interval between $x=X$ and $x=0$ is precisely one-quarter period or, the period may be determined from considering (3) and (5) in the time interval $[0, T / 4]$ and the space interval $[X, 0]$.

Now by Property (vi) of cosine-like functions, $x(t)$ is monotone in $[0, T / 4]$; therefore it possesses the inverse

$$
t=t(x, X)
$$

on the interval $[X, 0]$. The existence of this inverse is the essential property needed in the direct methods of constructing R-curves, and it is necessary in Rauscher's ingenious iteration method [2]. It is evident, that the existence of (6) is assured in the case considered here.

The substitution of (6) in (3) rcsults in

$$
\ddot{x}+f(x)=P g(x, X)
$$

where

$$
g(x, X)=g[t(x, X)]
$$

and it is well-known that (7) may be integrated in quadrature. One finds

$$
t(x, X)=\frac{1}{\sqrt{ } 2} \int_{X}^{x} \frac{d w}{\sqrt{[\varphi(w, X)-P \psi(w, X)]}}
$$

where

$$
\begin{gathered}
\varphi(x, X)=F(X)-F(x)=\int_{x}^{X} f(u) \mathrm{d} u \\
\psi(x, X)=G(X, X)-G(X, x)=\int_{x}^{X} g(u, X) \mathrm{d} u
\end{gathered}
$$

In analogy to elliptic integrals or Gamma and Beta-functions, one may regard (8) as an "incomplete" integral. Then, the complete integral gives the quarter-period, or

$$
T(X, P)=\frac{4}{\sqrt{ } 2} \int_{X}^{0} \frac{d w}{\sqrt{ }[\varphi(w, X)-P \psi(w, X)]}
$$


which is of the form

$$
T=T(X, P)
$$

Evidently, (11) is the equation of the R-curves because it relates period $T$ to amplitude $X$ with force amplitude $P$ as a parameter.

Actually (8) is an integral cquation of the form

$$
t(x, X)=\int_{X}^{x} H[t(w, X)] \mathrm{d} w
$$

because $\psi(w, X)$ depends explicitly on $t$. This is the property used in the method of Rauscher who iterates on this integral equation, beginning with the function $t_{0}(x, X)$ which he finds by putting $P=0$ in (8).

Our aim is, to find the differential equation which (11) satisfies, and to examine the incidence and character of its singular points.

\section{THE DIFFERENTIAL EQUATION OF R-CURVES}

In order to determine the differential equations of the R-curves, it is helpful to make use of the function

$$
T(X, 0)=T_{0}(X)=\frac{4}{\sqrt{ } 2} \int_{X}^{0} \frac{\mathrm{d} w}{\sqrt{[}[\varphi(w, X)]}
$$

which results from putting $P=0$ in (10) or (11). Clearly, this is the period of free vibrations as a function of the amplitude. It has been termed the "backbone" curve by Klotter [10].

Since resonance and dynamic vibration absorption exist for all $P>0$, if they exist at all, we may take $P$ to be small in (10). For small force amplitude, that equation may be solved explicitly for $P$ (by making use of the binomial theorem) and one finds

$$
P=\frac{N(T, X)}{D(X)}
$$

where the numerator

$$
N(T, X)=(\sqrt{ } 2) T+4 \int_{0}^{x} \varphi^{-\frac{1}{2}} \mathrm{~d} w
$$

and the denominator

$$
D=-2 \int_{0}^{x} \psi \varphi^{-\frac{3}{2}} \mathrm{~d} w
$$

Thus, (13) is the explicit solution

$$
P=P(T, X)
$$

for (11), when $P>0$ is sufficiently small.

Now, we wish to find the differential equation of (11) for $P=$ const., and along such a curve

$$
\mathrm{d} P=\frac{\partial P}{\partial X} \mathrm{~d} X+\frac{\partial P}{\partial T} \mathrm{~d} T=0
$$


and, from (17)

$$
\mathrm{d} T=-\frac{\partial P / \partial X}{\partial P / \partial T}
$$

where, from (11),

$$
\begin{aligned}
& \frac{\partial P}{\partial T}=\frac{\sqrt{ } 2}{D}, \\
& \frac{\partial P}{\partial X}=\frac{D \partial N / \partial X-N \partial D / \partial X}{D^{2}} .
\end{aligned}
$$

Substitution of these quantities in (18) gives the differential equation of the R-curves when $P$ is a small positive constant. It is

$$
(\sqrt{ } 2) \frac{\mathrm{d} T}{\mathrm{~d} X}=N \frac{\partial D / \partial X}{D}-\frac{\partial N}{\partial X}
$$

\section{THE SINGULAR POINTS}

By definition, the singular points of (19) are those points in the $X T$-plane for which $\mathrm{d} T / \mathrm{d} X$ does not exist. We first state:

Lemma I : Every singular point of (19) lies in the $T$-axis.

To prove it, we write (19) in the form

$$
\frac{\mathrm{d} T}{\mathrm{~d} X}=\frac{N \partial D / \partial X-D \partial N / \partial X}{D}
$$

where we have suppressed the unimportant factor $\sqrt{ } 2$. It follows that, at a singular point of (20), one must necessarily have either $D=0$ or $|D|=\infty$. We shall show that $D$ has no zeros for any bounded $X$, and that $D(0)=\infty$. That quantity is defined (except for a constant) by

$$
D(X)=\int_{0}^{X} \psi(w, X)[\varphi(w, X)]^{-\frac{3}{2}} \mathrm{~d} w
$$

and one sees from the definitions of $\psi$ and $\varphi$, that the integrand of (21) does not change its sign in the interval of integration and that it is not identically zero. Hence, $D(X) \neq 0$ for $X \neq 0$. This proves the first of the above assertions.

Now, $\psi$ and $\varphi$ are both monotone functions of $w$. Hence we may use the mean-value theorem and write (21) as

$$
D(X)=[\varphi(\xi, X)]^{-1} \int_{0}^{X} \psi \varphi^{-\frac{1}{2}} \mathrm{~d} w
$$

where $\xi$ lies in $0 \leqslant \xi \leqslant X$. Integration by parts of (22) gives

$$
D(X)=[\varphi(\xi, X)]^{-1}\left\{\left[\psi(X, X) T_{0}(X)-\psi(X, 0) T_{0}(0)\right]-\int_{0}^{X} T_{0}(w) g(X, w) \mathrm{d} w\right\}
$$


where use was made of the definition of $\psi$ given in (9), of the definition of $T_{0}(X)$ given in (12), and of

$$
\frac{\mathrm{d} G}{\mathrm{~d} w}=-g(X, w)
$$

which is an immediate consequence of (9). Now, in (23),

$$
\begin{aligned}
& \psi(X, X)=0, \\
& \psi(X, 0)=-G(X, 0) \neq 0, \\
& T_{0}(0) \neq 0,
\end{aligned}
$$

and the integral in (23) is not improper for bounded $X$. Therefore, $D(X)$ does not become unbounded for any bounded non-zero $X$. This completes the proof of Lemma I.

We now prove

Theorem II : Equation (19) has precisely one singularity. It lies at the intersection of backbone curve and $T$-axis, and it is a saddle point.

First we show that

$$
\lim _{X \rightarrow 0} D(X)=\infty
$$

In view of (21), this is not an intuitively obvious result. Inasmuch as $\xi$ of $(23)$ is in $[0, X]$, $\xi \rightarrow X$ in the limit as $X \rightarrow 0$. Thus, we proceed to the limit (25) in the sequence $\xi \rightarrow X \rightarrow 0$. But, from the definition of $\varphi$,

$$
\lim _{\xi \rightarrow X}[\varphi(\xi, X)]^{-1}=\infty
$$

Hence, if (23) is regarded to be of the form

$$
D=D(\xi, X)
$$

it turns out that

$$
\lim _{\xi \rightarrow X} D(\xi, X)=\infty
$$

even without proceeding to $X \rightarrow 0$. This proves (25). Next we demonstrate that, on the $T$-axis,

$$
\left.\frac{\partial N}{\partial X}\right|_{X=0}=0 .
$$

Since $f(u)$ is odd, $F(X)$ and $F(x)$ in (9) are even. This has the consequence that

$$
\varphi(X, x)=\varphi(-X, x)=\varphi(X,-x)=\varphi(-X,-x)
$$

and, therefore it is evident from (12) that

$$
T_{0}(X)=T_{0}(-X)
$$

Expressed differently, the $T$-axis is an axis of symmetry for the backbone curve. Thus, if the derivative $\mathrm{d} T_{0} / \mathrm{d} X$ exists for $X=0$, that derivative must itself vanish, or the backbone intersects the $T$-axis orthogonally. But, one sees from the definition (14) of $N$, that, except for a constant, 


$$
\frac{\partial N}{\partial X}=\frac{\mathrm{d}}{\mathrm{d} X} \int_{0}^{X} \varphi^{-\frac{1}{2}} \mathrm{~d} w=\frac{\mathrm{d} T_{0}}{\mathrm{~d} X}
$$

where the last equality results from (12). It follows, that (26) is true, and the differential equation (19) becomes, on the $T$-axis (i.e. for $X=0$ ),

$$
\frac{\mathrm{d} T}{\mathrm{~d} X}=\frac{N}{D /(\mathrm{d} D / \mathrm{d} X)}
$$

where we have already shown that $D$ in (28) is unbounded.

Now, it is easy to show that, on the $T$-axis,

$$
\frac{D}{\mathrm{~d} D / \mathrm{d} X}=0
$$

or, as $X \rightarrow 0, \mathrm{~d} D / \mathrm{d} X$ tends to infinity more rapidly that $D$ itself. If $D(X)$ is differentiable, we may write (by Taylor's formula) for $|X|$ sufficiently small

$$
D(X)=-X \frac{\mathrm{d} D}{\mathrm{~d} X}
$$

or

$$
X=-\frac{D(X)}{\mathrm{d} D / \mathrm{d} X}
$$

Thus, if $X$ can take on the value zero and $D(0)=\infty$ it follows that, as $X \rightarrow 0, \mathrm{~d} D / \mathrm{d} X \rightarrow \infty$ of a higher order than $D \rightarrow \infty$.

This result has an interesting interpretation, deducible from (28). By definition,

$$
N=N(T, X) \text {. }
$$

Then, if $N(T, 0) \neq 0$ is bounded, $\mathrm{d} T /\left.\mathrm{d} X\right|_{X=0}=\infty$ or, the $T$-axis is itself an integral curve of (28). But, in view of (11) $P=0$ when $D=\infty$ and $N \neq 0$ is bounded. Consequently, the $\mathrm{R}$-curve for $\mathrm{P}=0$ consists of the backbone curve and of the $T$-axis. By continiuty, then, the R-curves for $P>0$ sufficiently small lie in the neighborhood of the $T$-axis of and the backbone curve.

It follows from (29) that, at a singular point of (28), $N$ must either vanish or become unbounded on the $T$-axis. But, because of (12), the defining equation (14) may be written as

$$
N(T, 0)=(\sqrt{ } 2)\left[T-T_{0}(0)\right] .
$$

Here, $T$ is the period of the forcing function, and $T_{0}(0)$ is the period of free vibration at vanishingly small amplitude. $N(T, 0)$ cannot become unbounded for bounded $T$, and it has precisely one zero when the two periods coincide. This proves that the only singular point of (19) lies in the intersection of backbone and $T$-axis.

To complete the proof of Theorem II, it remains to show that the singularity is a saddle point. The simplest way of doing this is to consider (13) for $P=P_{0}$ and for $|X|$ sufficiently small. That equation is

$$
P_{0}=N / D
$$


To expand the numerator we use the form

$$
N(T, X)=(\sqrt{ } 2)\left[T-T_{0}(X)\right] .
$$

Therefore, except for a constant factor

$$
N=T-T_{0}^{\prime}(0) X+\ldots
$$

and we have already shown that, if $T_{0}^{\prime}(0)$ exists, it vanishes.

In expanding the denominator, we make use of the observation that, from its definition $\psi(x, X)$ is odd in $X$. Now, it was demonstrated in (25) that $[D(0)]^{-1}=0$. Hence, if the derivative $\mathrm{d} / \mathrm{d} X[D(X)]^{-1}$ exists for $|X|$ small, the expansion is necessarily of the form

$$
[D(X)]^{-1}=a X+\ldots
$$

where $a$ is a constant. Substituting (32) and (33) in (31), one has

$$
P_{0}=a T X+\ldots
$$

and this is the equation of a family of hyperbolas. In consequence, the singularity of (19) is a saddle point; this completes the proof of Theorem II.

\section{THE R-CURVE PORTRAIT}

One sees from (12) that $T_{0}(X)$ is a monotone function, and it has already been pointed out that

$$
T_{0}(X)=T_{0}(-X) .
$$

In consequence,

$$
N(T, X)=(\sqrt{ } 2)\left[T-T_{0}(X)\right]
$$

does not change its sign with $X$. However, it does have opposite sign according as $T>T_{0}$, or $T<T_{0}$. Moreover, $[D(X)]^{-1}$ is an odd function as was pointed out in connection with the expansion (33). Finally, one sees from (15) that $D<0$ when $X>0$. It follows that we may write (13) in the form

$$
P=(\sqrt{ } 2)\left[T_{0}(X)-T\right][X|D(X)| /|X|]^{-1}>0 .
$$

It is evident from this equation that $X$ must be replaced by $-X$ as $T$ goes from values $T<T_{0}$ to $T>T_{0}$, or $X$ changes its sign as $T$ passes through the value $T_{0}$.

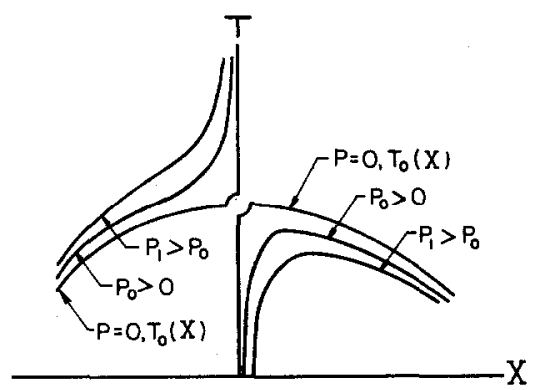

FiG. 3. Period amplitude plane. 
Finally, (10) shows that $T(X, P)$ is analytic in $P$. Consequently, two distinct R-curves $T_{1}=T\left(X, P_{1}\right)$ and $T_{2}=T\left(X, P_{2}\right),\left(P_{1} \neq P_{2}\right)$ cannot cross. It follows, that the R-curve portrait must have the appearance shown in Fig. 3. Their maps in the $X \omega$-plane and the $|X| \omega$-plane look as in Fig. 4, where $\omega=2 \pi / T$.
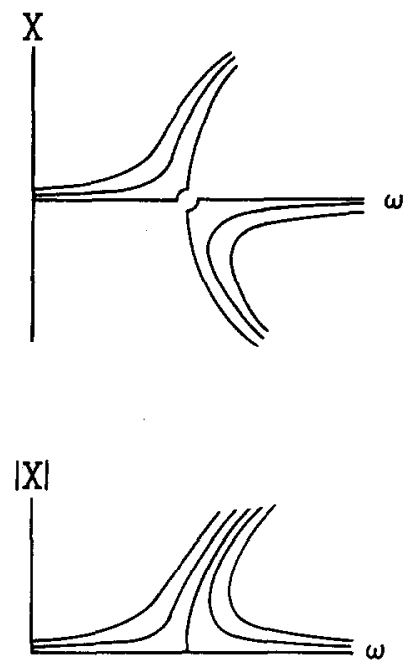

FIG. 4. Frequency-amplitude planes.

The diagrams of Fig. 4 have the appearance of those, usually constructed for the forced steady-state vibrations of weakly non-linear systems, subjected to simple harmonic excitation. Here we have shown, under what conditions the usually unquestioned construction of R-curves is justified when the system is not necessarily weakly non-linear, and when the periodic excitation is not simple harmonic.

Acknowledgement Support of this work from AFOSR Research Grant 1057-66 is gratefully acknowledged.

\section{REFERENCES}

[1] J. P. Den Hartog, Mechanical Vibrations, 3rd edition. McGraw-Hill (1956).

[2] M. RAuSCHER, Steady oscillations of systems with nonlinear and unsymetrical elasticity. J. appl. Mech. 5, (1938).

[3] J. J. STOKER, Nonlinear Vibrations. Interscience (1950).

[4] P. R. Sethna, Steady-state motion of one and two degrees of freedom vibrating systems with a nonlinear restoring force. Proc. 2nd U.S. Natn. Congr. Appl. Mech. The American Society of Mechanical Engineers (1954).

[5] T. C. HUANG, Harmonic oscillations of nonlinear two-degree-of-freedom systems. Trans. ASME, J. appl. Mech. 77, Ser. E (1955).

[6] W. KINNEY and R. M. RosenBERG, On steady-state harmonic vibrations of nonlinear systems with many degrees of freedom, J. appl. Mech. (to be published).

[7] W. D. KINNEY, On the geometrization of the forced oscillations of nonlinear systems having many degrees of freedom. Ph.D. Thesis, Berkeley, Cal. (1965).

[8] R. M. Rosenderg, On nonlinear vibrations of systems with many degrees of frecdom. Adv. appl. Mech. Academic Press (to be published). 
[9] W. SzempliŃSKa-STUPNICKA, Normal modes of a non-linear two-degrees of freedom system and their properties. Nonlinear Vibration Problems, Panstwowe Wydawnictwo Naukowe, Warsaw (1964).

[10] K. KLotTER, Steady-state vibrations in systems having arbitrary restoring and arbitrary damping forces. Proc. Symp. Nonl. Circ. Anal., Polytechnic Institute of Brooklyn (1953).

Résumé-Les notations suivantes sont définies : (i) fonction periodique ayant une amplitude, (ii) fonctions due type cosinus, (iii) vibrations forcées stationnaires. Les théorèmes suivants sont démontrés :

I. Soit

$$
\begin{gathered}
\ddot{x}+f(x)=P g(t)=P g(t+T), \\
x(0)=X \neq 0, \quad 0<P<\infty, \quad-\infty<t<\infty,
\end{gathered}
$$

où $g(t)$ est due type cosinus. Pour qu'il existe des vibrations stationnaires, il faut que $f(x)$ soit fonctions analytique, impaire et croissante de $x$, et que $x f(x)>0,(x \neq 0)$.

II. Les courbes de reponse des vibrations stationnaires $T=T(X, P)$ satisfont à une équation différentielle ordinaire du premier ordre qui a toujours une seule singularité au point $\left[X=0, T=T_{0}(0)=T(0,0)\right]$; celle-ci est un col.

En plus, on donne le portrait topologique des courbes de response.

Zusammenfassung--Die folgenden Begriffe werden definiert: (i) periodische Funktionen mit Amplitude, (ii) cosinusartige Funktionen, (iii) stationäre erzwungene Schwingungen. Es werden die folgenden Sätze bewiesen :

I. Es gelte

$$
\ddot{x}+f(x)=P g(t)=P g(t+T), \quad x(0)=X \neq 0, \quad 0<P<\infty, \quad-\infty<t<\infty,
$$

wobei $g(t)$ cosinusartig sei. Dann ist eine notwendige Bedingung für die Existenz von stationären Schwingungen, dass $f(x)$ eine analytische, ungerade, und mit $x$ steigende Funktion sei, und dass $x f(x)>0,(x \neq 0)$.

II. Die Vergrösserungsfunktionen (Resonanzkurven) $T=T(X, P)$ erfüllen eine gewöhnliche Differential gleichung erster Ordnung die genan ein Singulärstelle an $\left[X=0, T=T_{0}(0)=T(0,0)\right]$ hat; diese ist ein Sattelpunkt.

Ferner wird das topologische Portrait der Vergrösserungsfunktionen konstruiert.

Абстракт—Определяются следующие понятия : (i) непериодических функцин с амплитудои (ii) функции типа косинуса, (iii) установившегося состояния вынужденных колебаний. Доказываются следующие теоремы :

I. Если в задаче

$$
\ddot{x}+f(x)=P g(t)=P g(t+T), x(0)=X \neq 0,0<P<\infty,-\infty<t<+\infty,
$$

$g(t)$ является функциеи типа косинуса, то необходимое условие для существования состояния установившихся нолебании состоит в следующем : $f(x)$ должна быть функцией везде аналитической, нечетной, монотонно растущей $x$, причем $x f(x)>0,(x \neq 0)$.

II. Кривые соответствуюшие состоянию установившихся колебаний $T=T(X, P)$ удовлетворяют некоторому дифференциальному уравнению первого порядка с единственной особой точкой в $\left[X=0, T=T_{0}(0)=T(0,0)\right]$; эта точка является седловой.

Построена топологическая картина указанных кривых. 